\title{
Wahlen 2015 - Ihre Stimme zählt
}

\author{
Sandra Hügli
}

«Hausärzte Schweiz» publiziert vor den Eidgenössischen Wahlen Kurzinterviews mit Politikerinnen und Politikern aus der Ärzteschaft. Damit verbunden ist auch eine konkrete Wahlempfehlung unseres Verbandes.

Wir Haus- und Kinderärzte brauchen engagierte Parlamentarierinnen und Parlamentarier im Bund und in den Kantonen. Unterstützen Sie deshalb unsere Kandidatinnen und Kandidaten.

Wir wünschen allen politisch engagierten Hausärztinnen und Hausärzten viel Erfolg!

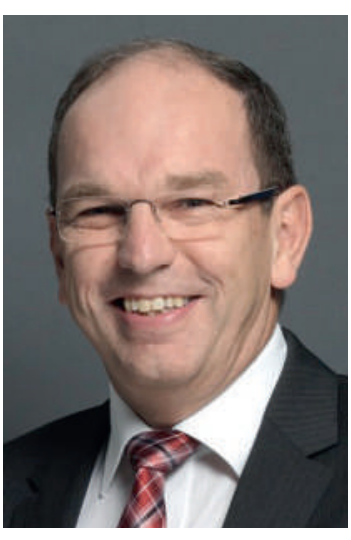

\section{Dr. Bruno Damann, CVP, St. Gallen}

Bruno Damann, geboren am 10.1.1957, verheiratet, Vater von 5 Kindern. Primarschule in Mörschwil SG. Gymnasium mit Matura Typ B im Kollegi Appenzell 1977. Medizinstudium zwischen 1978 und 1984 in Basel. Ausbildung als Grundversorger. Seit 1992 in einer Praxisgemeinschaft in Gossau. 2008 Gründung eines Ärztehauses in Gossau. 7 Jahre im Stadtparlament Gossau. Seit 2012 im Kantonsrat. Seit 2013 Stadtrat mit Teilzeitpensum in Gossau. Hobbies: Familie, Radfahren, Essen und Wein. Präsident des Gönnervereins Walter Zoo Gossau. J+S-Experte Leichtathletik. Verwaltungsrat der Sana Fürstenland AG Gossau. Verwaltungsratspräsident des Ärztehauses Lerchenstrasse Gossau AG. Verwaltungsratspräsident House of PORT AG. Mitglied Advisory Board HealthCare Information Bern (Galenica-Gruppe).

\section{Für welche politischen Schwerpunkte wollen Sie sich generell einsetzen?}

Gesundheitspolitik und Wirtschaft.

\section{Hausärzte/Hausärztinnen in der Politik - warum ist das wichtig?}

Es ist enorm wichtig, dass wieder mehr junge Menschen das Medizinstudium in der Schweiz absolvieren können. Dann muss die Ausbildung zum Hausarzt attraktiv gestaltet werden. Es müssen auch genügend Ausbildungsplätze für Hausärzte vorhanden sein. Der Beruf des Hausarztes muss wieder vermehrt gefördert werden. Dies kann man sicher in Bern besser beeinflussen, wenn man selber Hausarzt ist.

\section{Das Gesundheitswesen im Umbruch - welche Fragen und Themen beschäftigen Sie persönlich am stärksten?}

Mich beschäftigt vor allem die Frage nach genügend Nachwuchs in der Grundversorgung. Es wird wichtig sein, dass in Zukunft mehr Mediziner ausgebildet werden. Sonst werden wir noch mehr Mangel in der Grundversorgung haben.

Im Weitern sollten die administrativen Arbeiten nicht noch mehr ausgebaut werden. Auch die Kosten sollten wir langsam in den Griff bekommen. Als Grundversorger bin ich völlig gegen eine Staatsmedizin.

In der Ausbildung der jungen Medizinerinnen und Mediziner sollte man vermehrt auch den unternehmerischen Aspekt berücksichtigen.

Im Kantonsrat des Kantons St. Gallen werden wir uns bald mit der Vorlage zur Mediziner-Ausbildung befassen. Es wird wichtig sein, dass diese Vorlage erfolgreich sein wird.

\section{Die medizinische Grundversorgung - was bedeutet sie} Ihnen und wie sehen Sie die Zukunft der Grundversorger in der Schweiz?

Meiner Ansicht nach ist die Grundversorgung die erste Anlaufstelle bei gesundheitlichen Schwierigkeiten. Der Hausarzt ist auch aktiv in der Prävention. Wenn die Politik die Forderungen der Grundversorger umsetzt, dann sehe ich eine positive Entwicklung der Hausarztmedizin.

\section{Beschreiben Sie sich als Mensch in zwei Sätzen ...} Ich versuche bei Diskussionen zuzuhören und auf den Gesprächspartner einzugehen. Die Sache soll im Vordergrund stehen und nicht meine Person oder die Partei. 


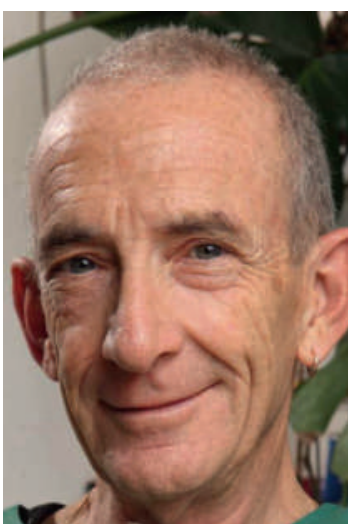

\section{Dr. David Winizki, Alternative Liste,} Zürich

Geboren 1948 als Drittgeneratiönler in Dietikon, religionslos, seit 1969 in der Stadt Zürich wohnhaft. Nach einer Erstausbildung zum Kaufmann Besuch eines Abendgymnasiums und Medizinstudium in Zürich bis 1979. Sozialisiert in der Aufbruchsstimmung der siebziger Jahre, folgten die stressigen Assistenzarztzeiten als Ehemann und junger Vater. 1989 Eröffnung einer hausärztlichen Doppelpraxis in Zürich mit starkem Anteil von lateinamerikanischen PatientInnen. Politisch immer schon aktiv in vielen gesundheitspolitischen Fragen, daneben in den neunziger Jahren für die Drogenlegalisierung und seit den Nullerjahren für die Gesundheitsversorgung von Sans Papiers, bringe ich meine Erfahrungen in der Alternativen Liste ein, deren Positionen von allen Parteien am ehesten mit meinen übereinstimmen.

Für welche politischen Schwerpunkte wollen Sie sich generell einsetzen?

Korrespondenz Sandra Hügli-Jost Kommunikationsverantwortliche «Hausärzte Schweiz», Geschäftsstelle Effingerstrasse 2

3011 Bern

sandra.huegli[at]

hausaerzteschweiz.ch
Je kleiner der Unterschied zwischen Arm und Reich ist, desto friedlicher ist die Gesellschaft und desto gesünder ihre Mitglieder, der Trend zur immer weiter klaffenden Schere muss mit allen Mitteln gestoppt und umgekehrt werden. Auch Umweltschutz ist ein dringendes Gebot der Stunde.

\section{Hausärzte/Hausärztinnen in der Politik - warum ist das wichtig?}

Wir HausärztInnen stehen beruflich inmitten verschiedener gesellschaftlicher Konflikte und fühlen dabei, dank unserer Vertrauensstellung, den Puls der Bevölkerung nicht nur am Vorderarm. Wir HausärztInnen sind in der Politik die Praktiker von der Front und bringen dadurch Inputs, die beachtet und respektiert werden.

\section{Das Gesundheitswesen im Umbruch - welche Fragen} und Themen beschäftigen Sie persönlich am

\section{Stärksten?}

Am meisten stört mich am Gesundheitswesen seine unsolidarische Finanzierung durch Kopfprämien. Dies führt zu Auswüchsen mit den Billigkrankenkassen (Assura), aber auch zu teuren administrativen Leerläufen wie der Prämiensubventionierung und anderem Übel mehr. Das Gesundheitswesen ist nicht zu teuer für die reiche Schweiz, sondern unsozial finanziert. Sinnlose ineffiziente Versuche die Kosten zu senken etwa mit der neuen Spitalfinanzierung - sind gar nicht nötig.

Die medizinische Grundversorgung - was bedeutet sie Ihnen und wie sehen Sie die Zukunft der Grundversorger in der Schweiz?

Das Gate-Keeper-Modell durch GrundversorgerInnen scheint mir die effizienteste Methode zur guten Gesundheitsversorgung der Bevölkerung zu sein. Die AkteurInnen sollen dafür gut ausgebildet und adäquat entgolten werden.

\section{Beschreiben Sie sich als Mensch in zwei Sätzen ...}

Ich bin ein überzeugter Hausarzt, der «seine» PatientInnen engagiert und parteiisch betreut, wie jeder gute Hausarzt. In den zwischenmenschlichen Beziehungen versuche ich, meinem Ziel einer möglichst herrschaftsfreien Gesellschaft zu entsprechen. 